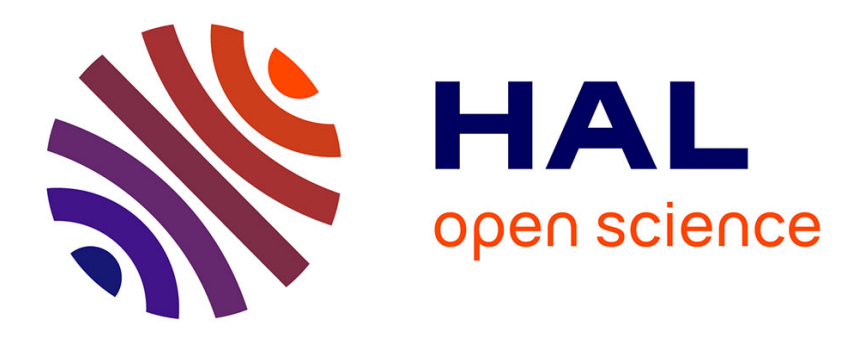

\title{
Une brève histoire du Département d'informatique de la PUC-Rio
}

\author{
Arndt von Staa, Jean-Pierre Briot
}

\section{To cite this version:}

Arndt von Staa, Jean-Pierre Briot. Une brève histoire du Département d'informatique de la PUC-Rio. Histoire de la recherche contemporaine: la revue du Comité pour l'histoire du CNRS , 2018, Dossier Histoire et structure de la coopération scientifique entre le Brésil et la France, VII (2), pp.168-179. hal-01840931

\section{HAL Id: hal-01840931 https://hal.sorbonne-universite.fr/hal-01840931}

Submitted on 12 Aug 2019

HAL is a multi-disciplinary open access archive for the deposit and dissemination of scientific research documents, whether they are published or not. The documents may come from teaching and research institutions in France or abroad, or from public or private research centers.
L'archive ouverte pluridisciplinaire HAL, est destinée au dépôt et à la diffusion de documents scientifiques de niveau recherche, publiés ou non, émanant des établissements d'enseignement et de recherche français ou étrangers, des laboratoires publics ou privés. 


\title{
Histoire de la recherche contemporaine
}

La revue du Comité pour l'histoire du CNRS

Tome VII N ${ }^{\circ} 2$ | 2018 :

Histoire et structure de la coopération scientifique entre le Brésil et la France Dossier: Histoire et structure de la coopération entre le Brésil et la France

\section{Une brève histoire du Département d'informatique de la PUC-Rio}

\author{
ARndt Von StaA
}

p. $168-179$

\section{Résumé}

Raconter l'histoire du département d’informatique de la PUC-Rio permet de revenir sur l'arrivée de l'informatique dans l'enseignement supérieur et la recherche au Brésil, et sur le rôle d'une université privée en particulier dans l'essor d'un domaine de pointe...

\section{Texte intégral}

La Pontifícia Universidade Católica do Rio de Janeiro (PUC-Rio) est une université privée jésuite qui a été créée en 1941. Elle fait partie d'un réseau de PUCs dans divers États brésiliens (Minas Gerais, Paraná, Rio Grande do Sul, São Paulo...) ainsi qu'en Amérique du sud, notamment au Chili. Bien que de taille modeste par son campus et son personnel (environ 1 ooo Professeurs et 18 ooo étudiants), elle a une réputation d'excellence et est la plus prestigieuse du réseau des PUCs. Son Département d'informatique a eu un rôle pionnier au Brésil, ayant été le premier créé dans l'histoire du Brésil et du fait de son rôle historique dans la formation de professeurs d'informatique d'autres universités. Il conserve une excellente réputation, son programme associé de post-graduation ${ }^{1}$ en informatique gardant depuis l'origine le meilleur classement ${ }^{2}$ par le programme d'évaluation triennal de la CAPES 3 . Ainsi, raconter l'histoire du département d'informatique de la PUC-Rio, c'est également un 
peu raconter l'histoire de l'arrivée de l'informatique dans l'enseignement supérieur et la recherche au Brésil.

\section{Genèse}

Tout a commencé lorsque la PUC-Rio, du fait de sa réputation d'excellence, a été choisie pour accueillir le premier ordinateur installé au Brésil. D’après les documents du Professeur Cardoso du Département de sciences des matériaux et de métallurgie (DCMM) de la PUC-Rio, qui était alors le président du $\mathrm{CNPq}^{4}$, un consortium a été formé par le CNPq, l'Institut de recherche de la Marine (IPqM) et le ministère des Affaires étrangères (Itamaraty), qui ont uni leurs forces pour amener en 1960 à la PUCRio un ordinateur Burroughs Datatron B-205. Il a été le premier ordinateur à être utilisé au Brésil à des fins scientifiques et a été installé dans le tout nouveau Centre de calcul des données (CPD) créé pour l'accueilli

3 Il s'agissait d'une machine respectable, pesant un peu plus d'une tonne et contenant environ 1600 valves doubles triodes et un énorme ensemble de diodes semiconducteurs, ainsi que des résistances et des condensateurs. Le tout consommait près de $30 \mathrm{kVA}^{5}$ de puissance. Pour qu'il puisse garder la tête froide et calculer correctement, le Centre de calcul des données est devenu le seul endroit de la PUC-Rio à bénéficier d'un système d'air conditionné. Apparemment l'air conditionné avait été bien configuré, du fait de la température agréable de la chapelle qui plus tard et jusqu'à maintenant occupe l'endroit où avait été installé le monstre.

4 La taille de la mémoire de l'ordinateur était - pour les normes de l'époque fantastique ! 4 ooo mots de 10 chiffres décimaux, c'est-à-dire environ 16 kilo octets (Ko). Les opérations étaient rapides, une addition était effectuée en o,1 milliseconde, dans les cas où nous pouvions produire un code capable de fonctionner dans la bande rapide de la machine, sinon cela prenait 1 milliseconde. Pour imprimer, on pouvait choisir entre une machine à écrire à marteaux et une imprimante tabulaire ultraefficace d'IBM, capable d'imprimer 60 lignes de 80 caractères numériques par minute. Cette imprimante tabulaire était en fait un ordinateur électromécanique, commandé par relais et programmé par le panneau de commande. Un panneau de commande était un type de carte en matériau isolant et rempli de trous. À travers des fiches placées introduites dans ces trous et connectées par des fils étaient alors établies des connections entre les relais et ainsi configuré le comportement de la machine.

5 L'ensemble des équipements valait environ un million de dollars américains de l'époque, aujourd'hui probablement près de 10 millions de dollars. Pour l'utiliser il était nécessaire de prendre rendez-vous, parfois plusieurs jours à l'avance. Pour les programmes de déverminage (" debugging »), on vérifiait dans la machine les instructions du programme, une par une, et ce faisant personne d'autre ne pouvait l'utiliser. Les résultats de l'exécution de chaque instruction étaient vérifiés à travers les innombrables lampes du panneau de commande. Bien entendu, tout téléphone portable moderne gagne de 1000 à zéro face à cette machine.

$6 \quad$ Le personnel du Centre de calcul des données (CPD) était constitué par les étudiants de la PUC-Rio, à l'exception du directeur, le Professeur Hélio Drago Romano, et d'un technicien de maintenance, Manoel Martins. Le Docteur Jacques Cohen, l'un des rares post-gradués au Brésil à l'époque a travaillé au CPD pendant un moment. Grâce à lui et au Père Amaral, beaucoup d'entre nous ont suivi leur premier cours de programmation au CPD à la PUC-Rio. Nous avons appris le b.a.ba du calcul informatique d'une manière bien plus frustrante qu'aujourd'hui, bien que les étudiants actuels ne le croient pas. Il y avait un professeur, Theodoro Oniga, qui donnait des cours de recherche opérationnelle qui comprenait des énigmes mathématiques. Peu survécurent au cours. Parmi ceux-ci, très peu ont osé continuer dans le métier informatique, à l'époque une voie pour les personnes un peu bizarres. Ensuite, le Pr Sergio Machado Rezende, à l'époque étudiant du cours d'ingénierie électronique de l'EPUC ${ }^{6}$ a pris soin des cours de programmation du B-205.

7 Divers programmes ont été développés au CPD. Certains des plus importants étaient coordonnés par le Professeur Jacques Cohen. Nous avons par exemple développé pour 
Furnas 7 , entre autres programmes, une simulation de fonctionnement de réservoirs hydroélectriques et une simulation de flux de charge. Le programme fonctionnait pendant des heures. Nous avons travaillé en équipe. Ceux qui ont passé la nuit au CPD pouvaient dormir dans une sorte de civière située derrière l'ordinateur. Si jamais des problèmes se produisent, par exemple la combustion d'une valve ou une résistance, une alarme sonnait avec un bruit infernal, réveillant n'importe qui de son sommeil mérité. À $22 \mathrm{~h}$ passées, l'unique garde faisait sa ronde et avertissait qu'il allait lâcher les chiens. Nous devions alors décider qui allait rester dormir derrière la machine, les autres allant dîner quelque part.

8 C'est là que j'ai rencontré le Professeur Carlos José Lucena en 1963. Lui, Carlos Valdesuso, Raphael Chrisóstomo Barbosa da Silva et d'autres composaient alors le Département de recherche opérationnelle (DPO), qui faisait partie du CPD. Parmi les nouveautés inventées par le groupe, on peut mentionner un prototype de compilateur du langage de programmation Algol. Comme le lecteur de carte ne savait pas lire les cartes perforées alphanumériques, ils ont inventé un moyen de lire par deux fois la masse de cartes, une fois à partir du haut, puis à partir du bas. Ensuite, il restait à traiter ce qui a avait été lu pour reconstituer les caractères alphabétiques. Je pense que cela mériterait un article décrivant ce programme.

$9 \quad$ En 1965, plusieurs universités, dont la PUC-Rio, ont reçu des ordinateurs IBM 1130. Ces ordinateurs, d'ores et déjà avec des circuits à faible intégration, avaient $8 \mathrm{Ko}$ de mémoire réelle et un disque de 5 Mo. Ils étaient dotés de deux choses très intéressantes : un système d'exploitation simplifié et un compilateur Fortran. Ceci a permis d'enseigner la programmation de manière plus large et sans douleur. Cette opportunité d'affaires - oui, nous étions aussi des entrepreneurs ! - a été identifiée par le Professeur Lucena, par Luiz Carlos Siqueira et par José Roberto Ribeiro dos Santos, qui ont décidé d'offrir un cours de calcul numérique à travers le Département de mathématiques récemment créé, alors dirigé par le Professeur Antonio Cesar Olinto. Quand je suis rentré d'un séjour d'un an et demi en cours de spécialisation en Allemagne, j'ai trouvé à la fin de 1967, ce cours en fonctionnement et avec plein de succès.

10 Pendant la période de mon absence, Antonio Carlos do Rego Gil et d'autres chez IBM ont négocié avec la PUC-Rio l'installation d'un ordinateur IBM 7044 en régime de prêt. Pour cela a été créé le Rio Data Centro (RDC). Le 7044 avait 32 Kmots de 36 bits (environ 180 Koctets) et fonctionnait avec un cycle de mémoire d'environ 6 microsecondes. Il était de la même famille que les ordinateurs 7094 encore largement utilisés dans les universités américaines, pourtant il existait des machines plus modernes comme le 1130 (très petit) et les premiers modèles d'IBM 360, entre autres modèles. La PUC-Rio avait à l'époque le plus grand centre de calcul scientifique au Brésil.

11 Pour savoir comment faire fonctionner cet ordinateur, le Professeur Lucena, Wilfried Probst et Luiz de Castro Martins, à l'époque analyste chez IBM, sont allés pendant l'hiver 1967 (de janvier à mars ${ }^{8}$ ) à Waterloo, au Canada. Là-bas, ils ont connu le Professeur Donald Cowan avec lequel le Professeur Lucena a établi une amitié étroite et féconde, qui a donné lieu à de nombreux articles publiés et à une collaboration intense avec le Computer Systems Group (CSG) de Waterloo, devenue connue là bas comme « la connexion brésilienne », du fait du nombre de Brésiliens y ayant étudié ou travaillé.

12 À travers la relation avec le Professeur Cowan s'est également mise en place une connexion avec le Professeur Calvin Gotlieb de l'Université de Toronto. Elle aussi a reçu un grand nombre d'étudiants post-gradués brésiliens.

13 Avec l'arrivée de l'ordinateur IBM 7044 s'est initiée une nouvelle phase. Il est devenu possible d'enseigner la programmation à tous les élèves du Centre Technique Scientifique (CTC) ${ }^{9}$, en introduisant le langage de programmation Fortran IV dès les premiers cours du cycle de base. Que nous nous sentions petits au moment d'entrer dans un auditorium rempli d'environ 200 étudiants ! Oui, en 1968 les classes du cycle de base avaient lieu dans deux auditoriums. Chaque classe avait environ 200 étudiants et, en général, il y avait deux classes en parallèle. En dépit de la quantité d'étudiants en classe, nous avons l'impression que les étudiants de l'époque apprenaient autant que les étudiants d'aujourd'hui. Nous avions alors la difficile tâche de les convaincre que la 
programmation était une discipline importante pour tout ingénieur et qu'un ingénieur qui ne saurait pas programmer finirait par avoir des difficultés à trouver un travail intéressant. Ils ont courageusement fait face à nos cours d'initiation à la science du calcul (informatique) et au calcul numérique. Par la suite ils ont pu résoudre leurs problèmes avec des règles de calcul, en ignorant pendant de nombreuses années l'existence des ordinateurs.

Pour donner aux étudiants une idée de ce qui se passait en détail dans la machine, nous avons développé un simulateur du vieux B-205, leur donnant ainsi une chance de produire des programmes dans un langage machine. Est ainsi né le cours PUC-007, enseigné pour plusieurs années en initiation à la science du calcul. Comme il était amusant de demander aux étudiants d'écrire dans ce simulateur des routines de conversion de nombre entier fractionnaire en nombre réel à virgule flottante ! Même ainsi, de nombreux étudiants en sont venus à aimer l'informatique - du moins c'est ce qu'ils me disent quand ils me voient.

\section{Période héroïque (1967 à 1970)}

Dans une des histoires du Baron de Münchhausen (un des meilleurs conteurs d'histoires extravagantes), il était à cheval dans un champ quand il est tombé dans un marécage. Comme il n'y avait personne pour l'aider et que les sables mouvants étaient déjà en train d'engloutir son cheval, Münchhausen a décidé de se sauver lui-même ainsi que son cheval. Il serra de toutes ses forces ses jambes autour du cheval et a commencé à tirer sur les lacets de ses bottes avec encore plus de force, jusqu'à réussir à s'extraire ainsi que son cheval du marais. De cette histoire est apparue l'expression « bootstrap » (auto-amorçage), largement utilisée en informatique et en général voulant dire développer quelque chose en utilisant cette même chose comme instrument. Cela vous semble extravagant ? Au contraire ! C'est en fait assez limpide. La création du programme de master du Département d'informatique a été essentiellement un processus d'auto-amorçage. Compte tenu des circonstances - la difficulté d'arriver à inviter des professeurs invités - le programme a été créé par lui-même, comme nous allons le voir.

Déjà en 1967, au Département de mathématiques, certains d'entre nous, dont les Professeurs Lucena, Furtado, Lins, Sérgio Carvalho, Luiz Martins, moi-même et quelques autres étions en même temps en train d'étudier et d'enseigner un nouveau master d'informatique à la PUC-Rio. Il s'agissait d'un programme dans lequel l'un de nous donnait un cours aux autres et nous tous tentions d'explorer ensemble tout ce qui était nouveau. Le nom du programme a été trouvé après une longue discussion, pour décider si nous devions adapter au Brésilien le terme Américain « computer science » ou bien le terme Français « informatique ». Le terme «informática » (informatique) a été choisi, en considérant que c'est un terme plus large. Est ainsi né le premier néologisme du domaine.

17 De curieux événements arrivaient, comme par exemple un étudiant soutenant sa thèse de master ayant pour directeur de thèse un « Professeur encadrant » qui ne l'avait lui-même pas encore soutenue. Phénomènes d'auto-amorçage... Sans cela, rien n’aurait pu être obtenu. Le courage était de mener un tel projet et la responsabilité était de veiller à ce qu'il ne tombe pas dans la médiocrité, mais au contraire qu'il devienne fort et respectable. Le Département d'informatique a été créé à la fin 1967 et a commencé à opérer de manière officielle en mars 1968, avec comme premier directeur le Professeur Olinto et comme premier coordinateur de post-graduation le Professeur Lucena. En mars 1968, ont commencé la première promotion régulière de master ainsi que la première classe du cours d'introduction à l'informatique et au calcul numérique, désormais devenu obligatoire pour tous les élèves du cycle de base du CTC.

Enfin, en 1969, tous ceux qui formaient le corps d'enseignants du Département d'informatique avaient conclu leur master, certains d'entre nous avec des unités de valeur obtenues dans des cours à l'Université de Waterloo. Nous pourrions passer à la seconde étape, l'obtention d'un doctorat. À l'époque il n'y avait pas encore l'exigence pour le corps enseignant de post-graduation d'être constitué de docteurs. Mais, dans la 
vision des Professeurs Olinto et Lucena, cela devait être l'objectif à atteindre par le département dès que possible. Le premier à aller suivre un doctorat à l'étranger était Sérgio Carvalho en 1969. En 1970, ce fut la deuxième vague et ainsi de suite, le master fonctionnant avec une régularité satisfaisante et produisant des personnels avec un bon niveau de formation.

\section{Phase de consolidation du master (1970 à 1975)}

Pendant une grande partie de la période 1970-1975, la plupart des professeurs étaient en doctorat à l'étranger. À la fin de cette période, quasiment tout le corps d'enseignants titulaires était formé de docteurs. Cette période a également été marquée par de grands changements dans le domaine de l'informatique. Le Brésil a lancé une "Politique nationale d'informatique » La communauté universitaire se sentait déjà assez forte pour constituer une société scientifique, la « Sociedade Brasileira de Computação » (SBC). Le Département d'informatique de la PUC-Rio a lui grandi et s'est consolidé.

En 1970, le Département d'informatique et le Rio Data Centro (RDC) ont obtenu un financement qui a permis la construction du bâtiment Rio Data Centro (RDC), qui a été amené à être utilisé par les deux à partir de $1972^{10}$. A également été négocié avec IBM l'installation d'un ordinateur IBM 370/165, un des plus grands ordinateurs disponibles à l'époque. Nous étions à nouveau en tête dans le pays, et peut-être aussi en Amérique latine, en terme de capacité de calcul informatique. Ont certainement contribué à cet accueil la crédibilité et la réputation acquises tant par le cours de master que par le RDC. Nous recevions des étudiants de toutes les parties du Brésil ainsi que du Chili. Une bonne partie du corps enseignant initial des principales universités Brésiliennes a obtenu un master dans le Département d'informatique de la PUC-Rio.

Les programmes de post-graduation en sciences et de la technologie au Brésil ont été rendus possibles par José Pelúcio Ferreira, alors à la BNDE, depuis devenue la BNDES $^{11}$, à travers le FUNTEC ${ }^{12}$ qu'il a créé et géré. En 1964, la PUC-Rio avait commencé le premier programme de master en génie mécanique, entre autres, sous les auspices du FUNTEC. D'après ce qui m'a dit à plusieurs reprises, c'est de ce programme qu'est sorti le premier étudiant avec master formé au Brésil.

Comme il arrive toujours au Brésil, les choses qui fonctionnent bien croissent, deviennent chaque fois plus visibles, et soudain les dirigeants de l'économie décident d' « aider à améliorer ». Dans ce cas, l' " amélioration » a été la fermeture du FUNTEC avant d'en avoir créé une alternative, avec comme un des résultats en 1971 le fait que les professeurs de post-graduation de la PUC-Rio n'ont pas reçu de salaire pendant plus de 6 mois. La situation a été résolue avec la création de la FINEP ${ }^{13}$ et l'approbation du " Projet FINEP », qui est venu soutenir l'ensemble du Centre Technique Scientifique (CTS) de la PUC-Rio ainsi que la COPPE de l'UFRJ ${ }^{14}$ pendant plus d'une décennie.

De plus, au cours de cette période le gouvernement a décidé de rationaliser l'utilisation des ordinateurs au sein des administrations du gouvernement fédéral et des entreprises publiques fédérales. À cette époque, les professeurs du Département de génie électrique de la PUC-Rio ont développé un concentrateur de données pour la SERPRO ${ }^{15}$. Avec cet équipement, au lieu de perforer des cartes, les documents fiscaux étaient transcrits directement sur des bandes magnétiques. Ce concentrateur, et plus encore le " Pato Feio ${ }^{16}$ développé à l'EPUSP ${ }^{17}$ entre 1971 et 1972, ont motivé le projet de développer un mini-ordinateur national. Le projet G10 a été lancé, avec l'objectif de créer le matériel (à l'EPUSP) et le logiciel (au Département d'informatique de la PUCRio) de cet ordinateur. Ce fut l'un des premiers projets « industriels » auxquels le département d'informatique a participé. Le projet a montré qu'il était possible et intéressant de combiner enseignement, recherche et développement. Malheureusement, en raison du manque de vision à long terme et d'intérêts immédiats (disposer d'une source de ressources pour faire face au manque de paiement des salaires), la conduite du projet s'est focalisée de plus en plus sur le développement, au détriment de l'enseignement et, certainement, de la recherche.

$24 \quad$ En 1975, après le retour des enseignants qui étaient partis pour obtenir leur doctorat et sous la direction du Professeur Lucena, le Département d'informatique a opté pour le 
côté académique de l'enseignement et de la recherche. En conséquence, le projet G1o et une partie significative du corps enseignant ont été transférés à l'entreprise d'état COBRA récemment créée, dont la mission était de développer et de commercialiser des ordinateurs et leurs logiciels de base. Au final, le projet G1o a évolué, devenant l'ordinateur COBRA-500, dont plusieurs unités furent commercialisées dans le pays. Ce fut, sans aucun doute, l'un des premiers exemples de succès de création de " spin-off », bien qu'involontaire et quelque peu traumatique.

$\mathrm{Au}$ début de « l'ère informatique » au Brésil, la formation à l'utilisation des machines, à de rares exceptions près, était faite par les fabricants eux-mêmes. Les cours en général étaient très appliqués, de faible qualité et ne traitaient pas de concepts. D’une certaine manière, les participants étaient formés à utiliser l'équipement, sans savoir ce qu'il y avait derrière le matériel et du logiciel qu'ils avaient appris à utiliser. Ceci a amené le Professeur Luiz Martins, alors Directeur du RDC, à créer le cours d'extension (pour un public non universitaire) en analyse de systèmes au RDC. Ce cours était indépendant d'un équipement spécifique et était axé sur les concepts et l'enseignement, plutôt que sur la simple formation. Ce cours a ensuite été transféré à la Coordination centrale d'extension (CCE) ${ }^{18}$ de la PUC-Rio, et est devenu pendant de nombreuses années, le cours phare de la CCE. On pourrait aller jusqu'à dire que c'est ce cours qui a viabilisé le CCE. Afin de parvenir à une plus grande exhaustivité, ce cours a ensuite été transformé en un cours de spécialisation en analyse de projet et gestion de systèmes, plus tard découplé en deux cours de spécialisation : un d'analyse de projets et un autre de gestion de projets.

Enfin, en raison des turbulences de l'époque, la « Politique nationale d'informatique » a été mise en place qui, du fait de l'interdiction d'importer du petit matériel à bas coût, a fortement entravé la recherche appliquée et le développement du génie logiciel.

\section{Phase de consolidation de doctorat (1975 à 1982)}

Le programme de master était déjà mûr et fonctionnait bien. Il y avait une forte demande par des futurs chercheurs ainsi que par des professionnels qui souhaitaient se recycler. Grâce au projet FINEP, les problèmes financiers du CTC et, par extension du Département d'informatique, avaient été résolus. Ainsi, maintenant que le corps enseignant était suffisamment qualifié, le Département d'informatique pouvait initier un programme de doctorat.

Les enseignements reçus à l'UCLA ${ }^{19}$ ont conduit le Professeur Lucena à nous encourager à produire des articles et établir des liens avec d'autres institutions nationales ou étrangères. Parmi ces relations, des liens ont été consolidés principalement avec l'Allemagne, à travers l'accord avec la GMD ${ }^{20}$, ainsi qu'avec le Canada, à travers l'accord avec la CIDA $^{21}$, cette dernière impliquant également des universités du Nord-Est du Brésil. Grâce à ces engagements, nous avons eu le plaisir d'accueillir de nombreux professeurs invités qui sont restés ici pendant plusieurs mois, parfois des années. Ces accords ont certainement beaucoup contribué à la maturité du Département d'informatique. Ils ont également contribué à ce que nos étudiants perdent leurs craintes sur la qualité et la compétitivité de nos cours.

Progressivement, nous avons commencé à publier de plus en plus d'articles nationaux et internationaux qui constitue la série de monographies en informatique du Département. Ces monographies étaient distribuées à diverses universités brésiliennes, et pour celles rédigées en anglais, également à une liste élargie à des universités étrangères.

Écrire des articles, établir des contacts, c'était très difficile à l'époque. Le Département avait un seul téléphone opéré par une secrétaire qui criait dans les couloirs à la recherche de la personne qui venait de recevoir un appel. Ce téléphone avait le tempérament d'une luciole, parfois il fonctionnait, parfois non. Le problème de téléphonie a été amélioré lorsque le RDC et le Département ont uni leurs forces pour déployer un central téléphonique avec une ligne directe et deux postes. La difficulté était devenue de savoir qui devrait répondre : la RDC ou le Département. 
31 Les articles étaient écrits en utilisant une machine à écrire. Les figures étaient dessinées à l'encre sur papier. Celle qui tapait les articles était une dactylo sans aucune connaissance de l'anglais. Les articles étaient dactylographiés à plusieurs reprises, chaque étape corrigeant certaines erreurs et en introduisant d'autres. Arriver au final à un texte de bonne qualité était une énorme bataille entre l'auteur et la dactylo. À la fin, ils ne pouvaient plus supporter de se regarder. Un ordinateur pour aider ? Pas la peine d'y penser. Une imprimante laser ? Elle était encore à inventer. Les machines Xerox existaient, mais il y en avait peu sur le campus et qui étaient de mauvaise qualité et difficiles d'utilisation. En résumé, on pourrait dire que la finition d'un article était un indicateur de sa qualité. Après tout, personne n'irait investir autant d'efforts et d'adrénaline pour donner une bonne finition à un article contenant des résultats faibles...

Avec le projet FINEP, il était devenu possible d'établir une bonne bibliothèque, ayant une collection significative d'ouvrages, un nombre important d'abonnements à des revues de renom et avec un programme d'échange de rapports techniques avec un bon nombre de bonnes universités étrangères. Nous avons convaincu le RDC de céder sa bibliothèque de manuels IBM au Département informatique. Est ainsi née, tout d'abord à une échelle modeste, ce qui allait devenir l'une des meilleures bibliothèques d'informatique du pays. Avec beaucoup d'efforts et quelques ruptures de continuité, nous avons réussi à la faire fonctionner jusqu'en 2013. Avec l'avènement du système d'accès à des revues via le portail Web Periódicos de la CAPES, de Google et du Kindie, disposer d'une bibliothèque physique avait perdu de sa pertinence. En conséquence, nous avons transféré notre bibliothèque à la bibliothèque centrale de la PUC-Rio.

Nous achetions des livres à cette époque. Première question : Quels livres intéressants ont été publiés ? Nous recevions des catalogues, mais peu convaincants. Avec quelles ressources ? Nous avions le projet FINEP, mais c'était limité. Il y avait des aides individuelles du CNPq, très utilisées pour l'achat de livres. Mais il n'y avait pas encore Amazon et importer quelque chose était une énorme difficulté. On devait recourir à une société qui utilisait le « dollar livre », environ le double du dollar dans le taux de change parallèle. Puis vint un colporteur de livres. Il était pire qu'un vendeur d'Encyclopédie universelle. Il ne quittait pas votre bureau avant de vous avoir vendu au moins un livre. Le plus étonnant, c'est qu'il savait exactement les sujets d'intérêt de chaque professeur et combien il avait encore dans son budget.

Participer à des congrès internationaux était également difficile, presque impossible. En vertu de certains accords, notamment avec IBM, le Département disposait de quelques ressources pour que nous puissions nous rendre à des congrès. De manière générale, c'était cependant une tâche très difficile d'arriver à convaincre certains mécènes (IBM, CNPq, CAPES ou même la PUC-Rio) de financer le voyage d'un chercheur à un congrès où il avait un article accepté.

\section{La phase initiale des micros (1982 à 1995)}

Vers 1980, le modèle du projet FINEP a commencé à montrer des signes d'épuisement. Les projets du CTC qui étaient auparavant retenus par la FINEP commençaient à être plus difficiles à être acceptés. L'exigence de rapports techniques et financiers devenait chaque fois plus fréquente. À un moment donné, nous avons tenté de diviser le projet global de la CTC en une série de projets propres à chaque département. Jusqu'à ce qu'au bout du compte le gouvernement réussisse à mettre le pays en faillite en 1982. Qui voulait voyager devait se rendre à la Banque du Brésil afin d'organiser une remise d'argent grâce à une banque installée à sa destination, mais la somme étant limitée à 2000 dollars américains par personne.

Il était nécessaire de faire appel à différentes agences de financement pour garantir la survie du Département d'informatique et l'intégrité du CTC. À l'époque il y avait une entreprise, ConsulPuc, établie au 5ème étage du bâtiment RDC, et dont la mission était de servir d'entité juridique responsable des projets menés par les professeurs. ConsulPuc était une société privée conventionnelle (à responsabilité limitée), ce qui rendait difficile la signature de contrats avec des entreprises publiques. José Pelúcio 
Ferreira et le Professeur Lucena ont alors créé la Fondation Padre Leonel Franca $(\mathrm{FPLF})^{22}$ à la place de ConsulPuc. La mission de la FPLF était et reste de servir d'intermédiaire aux projets entre les membres du corps professoral, la PUC-Rio et les entreprises. Mais, en tant que fondation, le FPLF est soumise à une législation fiscale et opérationnelle qui assure la transparence et, en outre, facilite la conclusion de contrats avec des entreprises publiques. L'un des avantages de la FPLF est de pouvoir transférer des ressources à la PUC-Rio ou un de ses départements et de viabiliser la création, la mise à jour et la maintenance de laboratoires, ainsi que l'embauche de chercheurs.

À cette époque, les premiers microordinateurs ont commencé à arriver. Il s'agissait en général d'engins assez volumineux, sans disque rigide, avec peu de mémoire et encore moins de logiciel. À travers des contrats individuels, nous en avons acheté un certain nombre. Ils étaient tous dans une même pièce. Plus tard, un espace de bibliothèque a été occupé puis une pièce légèrement plus grande a été choisie pour les accueillir.

38 L'Internet était encore en train de se traîner. La FPLF avait un microordinateur qui était connecté par ligne privée à un ordinateur du Laboratoire National de Calcul Scientifique $(\mathrm{LNCC})^{23}$. Celui-ci, à son tour, était connecté à un réseau capable de communiquer avec des ordinateurs de certaines des universités nationales ou étrangères. Chaque fois que l'on essayait d'utiliser ce réseau, il fallait prier avec ferveur pour que tout fonctionne bien. Malgré tout, il a été possible d'utiliser ce système pour participer à des activités coopératives, telles que la sélection des communications soumises à des congrès internationaux. C'était une grande nouveauté à l'époque, car auparavant tout était organisé par courrier, et se concluait par une réunion présentielle dans une ville à l'étranger. Bien sûr, le manque de ressources rendait toujours difficile, voire impossible, la participation à ces réunions.

Au fil du temps, le réseau de recherche brésilien s'est amélioré. Le CNPq a fait don de stations de travail Sun à diverses universités en vue de les interconnecter. On commençait à utiliser le courrier électronique, échangeant des messages avec d'innombrables autres institutions d'enseignement et de recherche éparpillées dans le monde entier.

40 Avec l'intermédiation du FPLF, ont surgi les premiers projets de recherche et de développement sont apparus. Ces projets ont montré qu'il était viable de concilier enseignement, recherche et développement, en assurant une fertilisation croisée.

Parmi ces projets, TecGraf, qui existe jusqu'à présent, est devenu un institut de la PUC-Rio et emploie plus de 200 employés, dont la plupart sont chercheurs docteurs. Les projets donnaient lieu à des articles, dissertations, thèses, jusqu'à des produits utiles. Les projets ont également permis l'installation de laboratoires modernes et très sophistiqués. Enfin, les projets ont permis d'engager des étudiants pour participer à des activités de développement selon une technologie de pointe. Ainsi une formule d'interaction université-entreprise a été mise en place qui n'entrait pas en conflit avec les intérêts de l'enseignement et de la recherche, comme cela avait été le cas pour le projet G1O.

\section{Stade de la participation dans la société de l'information (1995-1999)}

Les années 93 et 94 ont marqué un changement majeur dans la relation de la société avec les ordinateurs. Avant cela, les gens pouvaient avoir des microordinateurs à la maison ou au bureau. Ces ordinateurs pouvaient être connectés à des réseaux locaux, généralement très lents et pas toujours fiables. De plus, ces réseaux locaux n'étaient guère interconnectés avec d'autres réseaux. L'interconnexion transparente des réseaux n'existait que dans les universités et dans les instituts de recherche. À ce moment-là, il a été décidé de rendre l'Internet public.

43 Le Brésil a rapidement suivi la tendance en créant un Comité de pilotage d'Internet et en 1995 l'Internet public a commencé à exister au Brésil. Initialement, il a été considéré comme quelque chose réservé aux aficionados. Mais au fil du temps, son insertion dans la société est arrivée comme une avalanche. La conséquence a été la création d'un 
immense marché de services jusque-là impensables. Pourrait-on imaginer le monde actuel de l'enseignement, la recherche et le développement sans courrier électronique, sans transfert de fichiers, sans Google, sans Amazon, sans commerce électronique, réseau social ou autres ? Aujourd'hui, il est possible pour toute personne et partout dans le monde, dès qu'il dispose d'un ordinateur, un "smart phone » ou une tablette et d'une connexion suffisamment fiable et rapide, d'être aussi à jour qu'un chercheur dans un centre avancé d'enseignement et de recherche. Avant 1996, rien de cela n'existait ! Rien de tout cela n'était encore imaginable ? Eh bien, ce n'est pas vrai, parce qu'il a bien fallu que certains chercheurs et visionnaires puissent imaginer de telles innovations pour qu'elles puissent se matérialiser. Il est nécessaire d'aiguiser la créativité et il faut instruire les gens pour qu'ils puissent être créatifs.

La massification de l'utilisation des ordinateurs a entraîné une baisse importante du coût de l'équipement. Au début des années 1990, un micro-ordinateur avancé coûtait 6 ooo dollars ou plus. Aujourd'hui, des équipements beaucoup plus puissants peuvent être achetés pour 1200 dollars. Ceci, à son tour, a rendu les ordinateurs presque omniprésents. Rares sont maintenant les personnes de classe moyenne qui n'y ont aujourd'hui pas accès. Par voie de conséquence, de nouvelles formes d'organisation des entreprises sont apparues ainsi que de nouvelles façons d'organiser l'enseignement, la recherche, le développement et les loisirs.

En réaction à un appel à projets du CNPq et à l'initiative de José Alberto Aranha Sampaio et du Professeur Luiz Martins, le Département a participé en 1996 à un concours pour la sélection de projets d'institutionnalisation d'incubateurs d'entreprises d'informatique. Le projet Infogene a été sélectionné. Se posait alors le problème de l'endroit où l'héberger. Avant cela, l'Institut de Technologie de la PUC-Rio (ITUC) ${ }^{24}$ avait tenté de mettre en place un incubateur, l'Institut Genesis, avec les ressources pour construire un bâtiment, mais pas assez pour le rendre opérationnel. Avec le soutien de la FPLF, il a alors été proposé d'héberger Infogene dans l'incubateur Genesis et, en contrepartie, la FPLF s'est engagée à achever les travaux manquants. Aujourd'hui, l'incubateur Genesis est reconnu comme l'un des meilleurs du pays. Un signe de sa maturité est de souffrir depuis quelque temps du « mal de la PUC »- le manque d'espace physique pour grandir.

$46 \quad$ La conduite des projets de développement a également été étendue et améliorée. En réalité, la recherche de contrats de recherche est devenue nécessaire en raison de l'arrêt complet du projet FINEP. Au début, les professeurs du département ont regardé avec une certaine méfiance les quelques laboratoires thématiques menant des projets en partenariat avec des entreprises. Les deux premiers ont été l'ICAD et le TecGraf, coordonnés respectivement par les Professeurs Bruno Feijó et Marcelo Gattass. Puis nous avons commencé à y voir une excellente source de ressources pour les dépenses que la PUC-Rio avait du mal à couvrir. Les laboratoires du Département d'informatique ont été divisés en deux catégories : ceux d'enseignement et de recherche et ceux thématiques. Ces derniers devaient être autonomes et auto-suffisants, c'est-à-dire dont les dépenses liées à l'acquisition et l'entretien de matériel et de logiciel, paiement du personnel, construction et entretien des bâtiments devaient être financées avec des ressources que le laboratoire lui-même générait. De plus, une petite partie de ces ressources devait être transférée au Département, permettant ainsi l'évolution et la maintenance des laboratoires d'enseignement et de recherche. Cette formule a elle aussi été couronnée de succès. Aujourd'hui, le Département a une douzaine de laboratoires thématiques et pourrait peut-être en avoir plus, si nous ne souffrions pas du « mal de la PUC ».

47 Nous disposons ainsi aujourd'hui d'excellents laboratoires d'enseignement et de recherche. Les installations sont bonnes bien que l'espace soit réduit. Tout cela réalisé avec les ressources générées par les projets et par les cours de spécialisation coordonnés par le Département.

\section{Phase récente, recherche autour du Web (à partir de 1999)}


$48 \mathrm{Au}$ cours des dernières années, Internet s'est répandu et consolidé, devenant totalement intégré à la société. Internet a permis de nouvelles formes d'organisation des applications, avec des délais de livraison des logiciels de plus en plus courts, avec des exigences de qualité et une complexité des applications toujours croissantes. Comment attaquer et résoudre ces problèmes?

Certaines approches se basent sur des études théoriques et d'autres au contraire plus expérimentales. La deuxième option est souvent plus attrayante pour un jeune. Une question importante est de mener cela sans compromettre la qualité de l'enseignement et de la recherche.

50 Ce petit historique montre comment la création et le développement d'un département d'informatique a été conditionnée par les défis et surprises à la fois relatives au développement simultanément de l'informatique et de la science et la technologie au Brésil. Signalons que le texte original a été écrit en 2003. Depuis lors, le Département d'informatique et son programme de post-graduation associé continuent comme un des lieux d'excellence de l'informatique au Brésil. Notons que, notamment du fait de sa taille, il continue à ne pas aborder les aspects matériels (architectures matérielles et circuits imprimés). Les thématiques actuelles du Département et des laboratoires de recherche associés sont : base de données, génie logiciel, hypermedia, informatique coopérative et répartie, informatique graphique, interaction hommemachine, langages de programmation, optimisation, science des données et théorie.

\section{Bibliographie}

Cet article est une traduction et une adaptation par Jean-Pierre Briot du texte " HistóriaDepartamento de Informática - PUC-Rio » d'Arndt von Staa en 2003. Un texte plus détaillé sur l'installation du B-205 à la PUC-Rio est proposé par Margarida de Souza Neves, Silvia Ilg Byington et Arndt von Staa, « El B-205 en la PUC-Rio : Memoria y Historia de la computadora primera des amendes scientifiques en una Universidad brasileña ", Colloque d'histoire de l'informatique en Amérique latine et dans les Caraïbes, Medellin, Colombie, 2012.

\section{Notes}

1 Le programme de «post-graduation » («pós-graduação ») au Brésil, comprenant le Master et le Doctorat, a pour équivalent en France l'École doctorale. Un « post-gradué » est un étudiant ayant effectué une post-graduation, Master ou Doctorat.

2 Note 7, partagée au plus par 2 ou 3 programmes dans tout le pays pour une discipline donnée. Notez que les départements des universités ne sont pas évalués, par contre les programmes de post-graduation le sont, via une évaluation tous les 3 ans par la CAPES.

3 La Coordinação de Aperfeiçoamento de Pessoal de Nível Superior (CAPES) est l'agence fédérale d'évaluation et de financement de l'enseignement supérieur et de la recherche.

4 Le Conselho Nacional de Desenvolvimento Científico e Tecnológico, dont l'acronyme CNPq correspond à son nom original Conselho Nacional de Pesquisa, est l'agence fédérale de financement de la recherche scientifique.

5 Le kilo voltampère (abrégé en kVA) est une unité de mesure de la puissance électrique apparente.

6 École Polytechnique de l'Université Catholique (de la PUC-Rio).

7 La Furnas est une entreprise semi-publique de génération et de transport d'électricité.

8 Ce qui correspond également aux grandes vacances d'été au Brésil avant le début d'une nouvelle année universitaire, et ainsi à une disponibilité des professeurs.

9 Le « Centro Técnico Científico » (CTC) de la PUC-Rio agrège les enseignements et laboratoires de recherche de la PUC-Rio en sciences et en ingénierie.

10 Ce bâtiment, resté nommé RDC (Rio Data Centro), est toujours existant et est à ce jour occupé par le Département d'informatique.

11 La «Banco Nacional de Desenvolvimento Econômico e Social » (BNDES), Banque Nationale de Développement Économique et Social.

12 Le « Fundo Tecnológico » (FUNTEC), Fonds Technologique.

13 La «Financiadora de Estudos e Projetos » (FINEP), l'agence publique de financement des coopérations en $\mathrm{R} \& \mathrm{D}$ entre universités et industrie. 
14 L'« Instituto Alberto Luiz Coimbra de Pós-Graduação e Pesquisa de Engenharia », communément appelé la "COPPE », est la fédération de programmes de post-graduation en ingénierie de l'« Universidade Federal do Rio de Janeiro » (UFRJ).

15 L'entreprise publique leader pour les technologies de l'information pour le secteur public.

16 Le vilain petit canard.

17 L'« Escola Politécnica da Universidade de São Paulo ».

$18 \mathrm{La}$ « Coordinação Central d'Extensão » (CCE) de la PUC-Rio est le service de formation pour um public hors de l'université (entreprises, public, société civile...).

19 University of California, Los Angeles.

20 La « Gesellschaft für Mathematik und Datenverarbeitung », Société pour les mathématiques et le traitement des données, intégrée en 2001 à la Société Fraunhofer.

21 La «Canadian International Development Agency » (CIDA) était l'agence canadienne de développement international. Elle a depuis été fusionnée à l'intérieur du Département des affaires étrangères renommé « Global Affairs Canada ».

22 La « Fundação Padre Leonel Franca » (FPLF) a été nommée en hommage au fondateur et premier recteur de la PUC-Rio.

23 Un institut fédéral de recherche situé à Petrópolis, à environ une heure de Rio de Janeiro.

24 L'« Instituto Tecnológico da PUC-Rio » (ITUC) est en charge des prestations de services technologiques, tant de manière interne aux départements de la PUC-Rio que pour l'industrie et autres secteurs de l'économie.

\section{Pour citer cet article}

Référence papier

Arndt Von Staa, « Une brève histoire du Département d'informatique de la PUC-Rio », Histoire de la recherche contemporaine, Tome VII N² | 2018, 168-179.

Référence électronique

Arndt Von Staa, « Une brève histoire du Département d'informatique de la PUC-Rio », Histoire de la recherche contemporaine [En ligne], Tome VII N² | 2018, mis en ligne le 15 avril 2019, consulté le 10 août 2019. URL : http://journals.openedition.org/hrc/2416 ; DOI : 10.4000/hrc.2416

\section{Auteur}

\section{Arndt Von Staa}

Professeur émérite diplômé en ingénierie mécanique en 1965, docteur en informatique en 1969, Phd en 1974 à l'université de Waterloo. Académicien, commandeur de l'ordre du Mérite scientifique et technologique

Jean-Pierre Briot

Directeur de recherche CNRS, membre du laboratoire d'informatique de Paris 6 (LIP6), a dirigé le bureau du CNRS à Rio de Janeiro, et est chercheur invité permanent à l'Université PUC-Rio (Pontifícia Universidade Católica do Rio de Janeiro), dans le laboratoire de génie logiciel.

\section{Droits d'auteur}

(C) Comité pour l'histoire du CNRS 